\title{
Early Rapid Fluid Therapy Is Associated with Increased Rate of Noninvasive Positive-Pressure Ventilation in Hemoconcentrated Patients with Severe Acute Pancreatitis
}

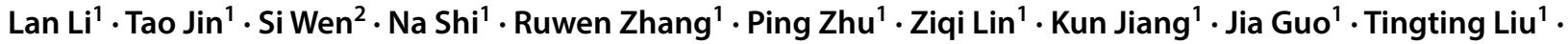 \\ Anthony Philips ${ }^{3} \cdot$ Lihui Deng $^{1} \cdot$ Xiaonan Yang ${ }^{1} \cdot$ Vikesh K. Singh $^{4} \cdot$ Robert Sutton $^{5} \cdot$ John A. Windsor ${ }^{6} \cdot$ Wei Huang $^{1}$. \\ Qing Xia ${ }^{1}$
}

Received: 15 September 2019 / Accepted: 27 November 2019 / Published online: 7 January 2020

(c) The Author(s) 2020

\begin{abstract}
Background/Aims Hematocrit is a widely used biomarker to guide early fluid therapy for patients with acute pancreatitis (AP), but there is controversy over whether early rapid fluid therapy (ERFT) should be used in hemoconcentrated patients. This study investigated the association of hematocrit and ERFT with clinical outcomes of patients with AP.

Methods Data from prospectively maintained AP database and retrospectively collected fluid management details were stratified according to actual severity defined by revised Atlanta classification. Hemoconcentration and "early" were defined as hematocrit $>44 \%$ and the first $6 \mathrm{~h}$ of general ward admission, respectively, and "rapid" fluid rate was defined as $\geq 3 \mathrm{ml} /$ $\mathrm{kg} / \mathrm{h}$. Patients were allocated into 4 groups for comparisons: group A, hematocrit $\leq 44 \%$ and fluid rate $<3 \mathrm{ml} / \mathrm{kg} / \mathrm{h}$; group $\mathrm{B}$, hematocrit $\leq 44 \%$ and fluid rate $\geq 3 \mathrm{ml} / \mathrm{kg} / \mathrm{h}$; group C, hematocrit $>44 \%$ and fluid rate $<3 \mathrm{ml} / \mathrm{kg} / \mathrm{h}$; and group D, hematocrit $>44 \%$ and fluid rate $\geq 3 \mathrm{ml} / \mathrm{kg} / \mathrm{h}$. Primary outcome was rate of noninvasive positive-pressure ventilation (NPPV). Results A total of 912 consecutive AP patients were analyzed. ERFT has no impact on clinical outcomes of hemoconcentrated, non-severe or all non-hemoconcentrated AP patients. In hemoconcentrated patients with severe AP (SAP), ERFT was accompanied with increased risk of NPPV (odds ratio 5.96, 95\% CI 1.57-22.6). Multivariate regression analyses confirmed ERFT and hemoconcentration were significantly and independently associated with persistent organ failure and mortality in patients with SAP.
\end{abstract}

Conclusions ERFT is associated with increased rate of NPPV in hemoconcentrated patients with SAP.

Keywords Acute pancreatitis · Fluid therapy $\cdot$ Hemoconcentration $\cdot$ Noninvasive positive-pressure ventilation $\cdot$ Persistent organ failure

Electronic supplementary material The online version of this article (https://doi.org/10.1007/s10620-019-05985-w) contains supplementary material, which is available to authorized users.

Qing Xia

xiaqing@medmail.com.cn

Wei Huang

dr_wei_huang@scu.edu.cn

1 Department and Laboratory of Integrated Traditional Chinese and Western Medicine, Sichuan Provincial Pancreatitis Center and West China-Liverpool Biomedical Research Center, West China Hospital, Sichuan University, No. 37 Wannan Guoxue Alley, Chengdu 610041, Sichuan Province, China

2 Department of Endocrinology and Metabolism, Yichang Hospital of Traditional Chinese Medicine, Yichang, China
3 Applied Surgery and Metabolism Laboratory, School of Biological Sciences, University of Auckland, Auckland, New Zealand

4 Division of Gastroenterology, Pancreatitis Center, Johns Hopkins Medical Institutions, Baltimore, USA

5 Liverpool Pancreatitis Research Group, Royal Liverpool University Hospital and Institute of Translational Medicine, University of Liverpool, Liverpool, UK

6 Surgical and Translational Research Center, Faculty of Medical and Health Sciences, University of Auckland, Auckland, New Zealand 


\section{Introduction}

The severe form of acute pancreatitis (SAP), defined as single or multiple persistent organ failure (OF) by Revised Atlanta Classification (RAC), carries a mortality of $36-50 \%$ [1]. The most common OF is respiratory dysfunction and failure [2,3]. Despite many advances in our understanding of the pathophysiology of AP, there remains no effective pharmacological treatment [4]. Fluid resuscitation is considered the cornerstone of early treatment of AP [5, 6], although there are many unanswered questions regarding the type of fluid to use, how fast to give it, and how best to monitor the response to it [7].

A number of studies have investigated the rate of fluid administration in patients with AP. Many clinicians advocate $[5,6]$ "early aggressive" fluid therapy on the basis that this reduces the systemic inflammatory response syndrome (SIRS), incidence of OF and hospital length of stay (LOS), but the benefits were more pronounced for mild than severe cases [8]. Other studies have suggested that in patients with predominately mild AP, early aggressive fluid therapy is associated with reduced $\operatorname{LOS}[9,10]$ and improved composite clinical outcomes [11]. In contrast, clinicians advocating a more moderate approach to fluid resuscitation argue that pancreatic necrosis is irreversible and that a higher volume of fluid given over the first $24 \mathrm{~h}$ is associated with higher incidence of respiratory dysfunction/failure, local complications, sepsis, and mortality [3, 12-14]. A randomized controlled clinical trial of patients with predicted SAP had a significantly worse clinical outcome with rapid fluid expansion $[15,16]$. The International Association of Pancreatology (IAP)/American Pancreatic Association (APA) [6], American College of Gastroenterology (ACG) [5], and Japanese Practice Guidelines [17] all recommend aggressive hydration although at different fluid rates (Table 1). Given the conflicting low level evidence, the American Gastroenterology Association (AGA) Practice Guidelines [18] only recommend "early goal-directed" fluid therapy without providing details for other important aspects of fluid therapy. While there is some evidence that early goal-directed therapy decreases mortality in severe sepsis and septic shock [19], subsequent trials have not confirmed this (i.e., ARISE, PROCESS, and PROMISE) [20-22]. Many other goals have been proposed [23] and while the goal-directed approach is an attractive concept, none have been adequately validated in the management of patients with AP. In the absence of a proven goal for fluid resuscitation, it is not yet possible to recommend a goal-directed approach in AP patients.

The most commonly used goals of fluid resuscitation are the normalization of urine output and blood pressure [23], but this has been shown to risk fluid overload and increased mortality [24]. The laboratory marker that shows promise as a goal and a guide to fluid therapy in patients with AP is hematocrit [25]. Hematocrit is the volume percentage of red blood cells in blood, or a marker of hemoconcentration or hemodilution. Hemoconcentration in AP was first described in the 1960s [26]. In 1998, Baillargeon et al. [27] reported that an admission serum hematocrit level $\geq 47 \%$ that did not decrease during the first $24 \mathrm{~h}$ was predictive of acute necrotic collection (ANC). Subsequent prospective studies [28-30] by the same group suggested that an admission hematocrit level $\geq 44 \%$, especially if it increased at $24 \mathrm{~h}$, was a significant predictor for both ANC and OF. This has been confirmed in a recent study of 1,612 AP patients, where admission hematocrit $>44 \%$ was a more accurate predictor of ANC and persistent OF than other biomarkers [31]. A randomized controlled clinical trial of patients with predicted SAP had a significantly worse clinical outcome when hematocrit was decreased to $<35 \%$ within $48 \mathrm{~h}$ of commencing fluid resuscitation, i.e., rapid hemodilution $[15,16]$. The IAP/APA Practice Guidelines recommend that the goal of fluid therapy should be to maintain hematocrit levels at 35-44\% [6].

In this study, we undertook retrospective analysis of prospectively collected data from the largest AP tertiary center in China (Supplementary Fig. S1) to determine the relationship between early rapid fluid therapy (ERFT), hemoconcentration, and clinical outcomes in patients with

Table 1 Summary of clinical guidelines of aggressive hydration in acute pancreatitis

\begin{tabular}{ll}
\hline Guidelines & Fluid rate \\
\hline 2013 IAP and APA & Goal-directed intravenous fluid therapy with 5-10 ml/kg/h \\
2013 ACG & Aggressive hydration, defined as $250-500 \mathrm{ml} / \mathrm{h}$ crystalloid solution \\
2015 Japanese guideline & Short-time rapid fluid resuscitation $(150-600 \mathrm{ml} / \mathrm{h})$ depending on the presence of shock and the dehydration level \\
2018 AGA & Goal-directed therapy for fluid management \\
2019 WSES & $\begin{array}{l}\text { Fluid overload is known to have detrimental effects, and early fluid resuscitation should be guided by frequent } \\
\text { reassessment of the hemodynamic status }\end{array}$
\end{tabular}

IAP International Association of Pancreatology, APA American Pancreatic Association, ACG American College of Gastroenterology, AGA American Gastroenterology Association, WSES World Society of Emergency Surgery 
AP after general ward admission. The hypothesis was that ERFT is associated with increased risk of respiratory dysfunction requiring noninvasive positive-pressure ventilation (NPPV) in hemoconcentrated SAP patients.

\section{Materials and Methods}

\section{Study Design and Setting}

A retrospective analysis was conducting using the STROBE guidelines [32] of the AP database at West China Hospital of Sichuan University. This is the largest center in China managing patients with AP. The study protocol was approved by the Institutional Review Board (No. 247) of the hospital, and informed consent was waived. Data from consecutive AP patients admitted to the Pancreatitis Center between January 1, 2016, and August 31, 2017, were prospectively collected into our database (Microsoft Access, Redmond, USA) by data managers using electronic Heath Information System (Donghua Medical, Shenzhen, China). Data regarding fluid therapy were retrospectively collected using International Classification of Diseases 10th edition (ICD-10) code K85 for patient identification.

\section{Data Collection}

Quality assurance, quality control, standard operating procedures, and researcher training were implemented in the study process. Retrospective data collection for fluid collection was undertaken by two experienced clinical researchers (LL and $\mathrm{TJ}$ ) using a pre-defined pro forma designed by senior authors (QX and WH) and audited by clinical leads (LD, $\mathrm{KJ}$, and ZL).

\section{Inclusion and Exclusion Criteria}

AP was diagnosed by two of three criteria: epigastric abdominal pain, elevated amylase or lipase $>3$ times of the upper limit of normal, and/or imaging consistent with AP [1]. Confirmed AP patients who had symptom onset $\leq 48 \mathrm{~h}$ when admitted to general wards were included regardless of the disease episodes. Exclusion criteria included those in whom AP was not the primary admitting diagnosis; ages $<18$ or $>70$ years; patients transferred to ICU admission within $24 \mathrm{~h}$ of ward admission; AP due to trauma or pancreatic cancer; chronic pancreatitis; pregnancy; advanced comorbidities; incomplete data for extraction (for example, lack of body weight, height, or computed tomography images).

\section{Fluid and Intensive Care Management}

In the West China Hospital, AP patients are initially admitted to the Emergency Department (ED). After confirming the diagnosis and initiation of fluid therapy with normal saline in the ED, patients are transferred to a general ward. Once transferred to the ward, patients have continuation of fluid therapy with (normal saline or Ringer's lactate solution) at $1-5 \mathrm{ml} / \mathrm{kg} / \mathrm{h}$ for the first $24 \mathrm{~h}$, with the rate based on predicted severity of AP and the clinical assessment of hydration status, rather than based on a pre-defined resuscitation protocol. Patients do not routinely receive additional fluid as a bolus (e.g., $10-20 \mathrm{ml} / \mathrm{kg} / \mathrm{h}$ over $30-45 \mathrm{~min}$ ) unless they were shocked, with mean arterial pressure $<65 \mathrm{~mm} \mathrm{Hg}$ [33]. Patients are admitted to a ward-based high-dependency unit (HDU) if they meet the criteria for NPPV [34]. Indications for NPPV are: patients alert and orientated, able to control airway and clear secretion but experiencing tachypnea (respiratory rate $>25 / \mathrm{min}$ ) or dyspnea (use of accessory muscles for breathing), or arterial partial pressure of $\mathrm{PaO}_{2}<60 \mathrm{~mm}$ $\mathrm{Hg}$, with or without partial pressure of $\mathrm{CO}_{2}>45 \mathrm{~mm} \mathrm{Hg}$ (measured by Roche Cobas b123 automatic blood gas analyzer; Shanghai, China), despite high flow oxygen by humidified non-rebreather mask (1060, Teleflex Medical; Limerick, PA, USA) at $8 \mathrm{~L} / \mathrm{min}$. The NPPV is delivered by Respironics V60 in the BiPAP S/T mode (Philips Respironics, BiPAP ${ }^{\circledR}$ Vision ${ }^{\mathrm{TM}}$; Pittsburgh, PA, USA) with a positive end expiratory pressure of 4-6 $\mathrm{cmH}_{2} \mathrm{O}$. Patients are admitted to intensive care unit (ICU) for mechanical ventilation, renal replacement therapy, and/or the need for continuous vasopressors/inotropes.

\section{Study Definitions}

"Severity" of AP The actual severity of AP patients was used to stratify patients, using the RAC criteria [mild AP (MAP), moderately severe AP (MSAP), and SAP] based on the most severe grade during period of index hospital admission [1].

"Early" fluid therapy This was the "first 6 h" of fluid therapy administered on the general wards, after transfer from ED. This did not include the fluid administered in the ED prior to transfer.

"Rate" of fluid therapy The rate of fluid infusion was defined as "rapid" if it was $\geq 3 \mathrm{ml} / \mathrm{kg} / \mathrm{h}$ (with "slow" $<3 \mathrm{ml} / \mathrm{kg} / \mathrm{h}$ ), based on the average rate of fluid therapy during the first $6 \mathrm{~h}$ of admission on general ward. Thus, ERFT would be $\geq 225 \mathrm{ml} / \mathrm{h}$ for a $75-\mathrm{kg}$ person over the first $6 \mathrm{~h}$ and "early slow fluid therapy" (ESFT) $<225 \mathrm{ml} / \mathrm{h}$. The cut-off fluid rate of $3 \mathrm{ml} / \mathrm{kg} / \mathrm{h}$ was taken from ACG Guidelines ( $\geq 250 \mathrm{ml} / \mathrm{h}$ ) [5]. 
Hemoconcentration Hematocrit was also measured at the time of admission to the general ward and not admission to ED. Hemoconcentration was defined as serum hematocrit $>44 \%[27,28,31]$.

\section{Patients Groups}

The patients were stratified into 4 groups based on hematocrit and fluid rate and all in reference to the first $6 \mathrm{~h}$ of admission to the general wards:

Group A hematocrit $\leq 44 \%$ on admission and fluid rate $<3 \mathrm{ml} / \mathrm{kg} / \mathrm{h}$,

Group B hematocrit $\leq 44 \%$ on admission and fluid rate $\geq 3 \mathrm{ml} / \mathrm{kg} / \mathrm{h}$,

Group C hematocrit $>44 \%$ on admission and fluid rate $<3 \mathrm{ml} / \mathrm{kg} / \mathrm{h}$,

Group D hematocrit $>44 \%$ on admission and fluid rate $\geq 3 \mathrm{ml} / \mathrm{kg} / \mathrm{h}$.

\section{Outcomes}

The primary clinical outcome measure was the rate of NPPV. We have selected NPPV as the primary endpoint because respiratory dysfunction/failure is the most common organ system affected, and because we have demonstrated that the most frequently noted consequence of ERFT is respiratory dysfunction [24]. In our setting, NPPV is the most commonly used means of organ support and it is frequently used in the general ward setting, and has been shown to reduce the need for ICU admission and invasive ventilation [34]. Secondary outcomes included persistent OF, multiple OF (MOF), HDU admission, ICU admission, local pancreatic complications, drainage necrosectomy, overall mortality, length of overall hospital stay (LOS), and costs. Definitions for etiologies and other study variables are shown in Supplementary Methods.

\section{Statistical Analysis}

Baseline parameters and clinical outcomes were compared between groups A and B (non-hemoconcentrated: ESFT vs. ERFT) or groups $\mathrm{C}$ and D (hemoconcentrated: ESFT vs. ERFT). Groups A and C (ESFT: non-hemoconcentrated vs. hemoconcentrated) were also compared with groups B and D (ERFT: non-hemoconcentrated vs. hemoconcentrated). Categorical data are expressed as number and percentage and compared by $\chi^{2}$ test (or Fisher's exact test). Continuous data are presented as median and interquartile range (IQR) and compared using Mann-Whitney $U$ test (2 groups) or Kruskal-Wallis $H$ test (3 groups) if distribution was skewed. Baseline variables with or without clinical severity score on ward admission were compared between groups using univariate analysis. Those covariates with $P$ value $<0.2$ were further fitted into: (1) multivariate logistic regression analyses for categorical clinical outcomes and linear regression analyses for qualitative clinical outcomes and LOS of MAP and MSAP [1], respectively, and expressed as odds ratio (OR) with 95\% confidence interval (CI); or (2) Cox regression analyses for predicted hospital discharge and expressed as hazard ratios (HR) with 95\% CI. In the Cox analysis, days for deceased patients are considered as truncated data. A two-sided $P<0.05$ was considered statistically significant. Statistical analyses were performed using SPSS ${ }^{\circledR} 21.0$ (IBM, Armonk, New York, USA).

\section{Results}

\section{Characteristics of Patient Cohort}

The algorithm for fluid therapy and patient selection is presented in Fig. 1. A total of 912 patients were included in the study. The baseline characteristics and clinical outcomes stratified by RAC severity grades [1] are summarized in Table 2: 352 (38.6\%) were MAP, 412 (45.2\%) MSAP, and $148(16.2 \%)$ SAP. The median age was 45 years (range 19 to 70 years), and $68 \%$ were males. Hypertriglyceridemia (36.7\%) was the leading etiology, followed by biliary (23.1\%) and alcohol (7.8\%), consistent with our recent findings from large cohorts $[2,35,36]$. The median time from symptom onset to general ward admission was $24 \mathrm{~h}$ (range 6-48 h), and that from ED to general ward was $6.5 \mathrm{~h}$ (range 4-19.5 h). Of 148 patients with persistent OF, 95 (64\%) had an onset $\leq 24 \mathrm{~h}$ of admission, followed by 44 (29.7\%), 3 (2\%) and $6(4 \%)$ on day 1,2 , and 3 and days $\geq 4$, respectively (Supplementary Fig. 2). There were 145 [15.9\%] respiratory, 10 [1.0\%] circulatory, and 22 [2.4\%] renal persistent OF with 22 (2.4\%) had MOF. There were 157 (17.2\%) admitted to HDU/ICU. Local pancreatic complications were diagnosed in a total of 518 patients: $331(46.5 \%)$ had acute peripancreatic fluid collections (APFC), and 187 (20.5\%) had ANC. Pancreatic necrosectomy was performed in $43(6.0 \%)$ patients. All 27 patients who died had SAP (27/148, 18.2\%), giving an overall mortality rate of $3.8 \%$ (Table 2 ).

\section{Baseline Parameters and Clinical Outcomes}

Patients with hemoconcentration on admission $(n=313)$, compared with those without hemoconcentration $(n=599$; Supplementary Table 1), were significantly younger (median 43 vs. 46 years, $P<0.001)$, more likely to be male $(86.6 \%$ vs. $58.2 \%, P<0.001)$, and had increased body mass index (26 vs. $24.7 \mathrm{~kg} / \mathrm{m}^{2}, P<0.001$ ), Charlson comorbidity index (median score 2 vs. $1, P<0.05$ ), and were associated with more severe clinical severity scores (SIRS, Glasgow, Acute 
Fig. 1 Fluid therapy and patient selection process of acute pancreatitis. Patients selection process of the study. $A P$ acute pancreatitis, MAP mild acute pancreatitis, $M S A P$ moderate severe acute pancreatitis, $S A P$ severe acute pancreatitis. *Initial fluid therapy

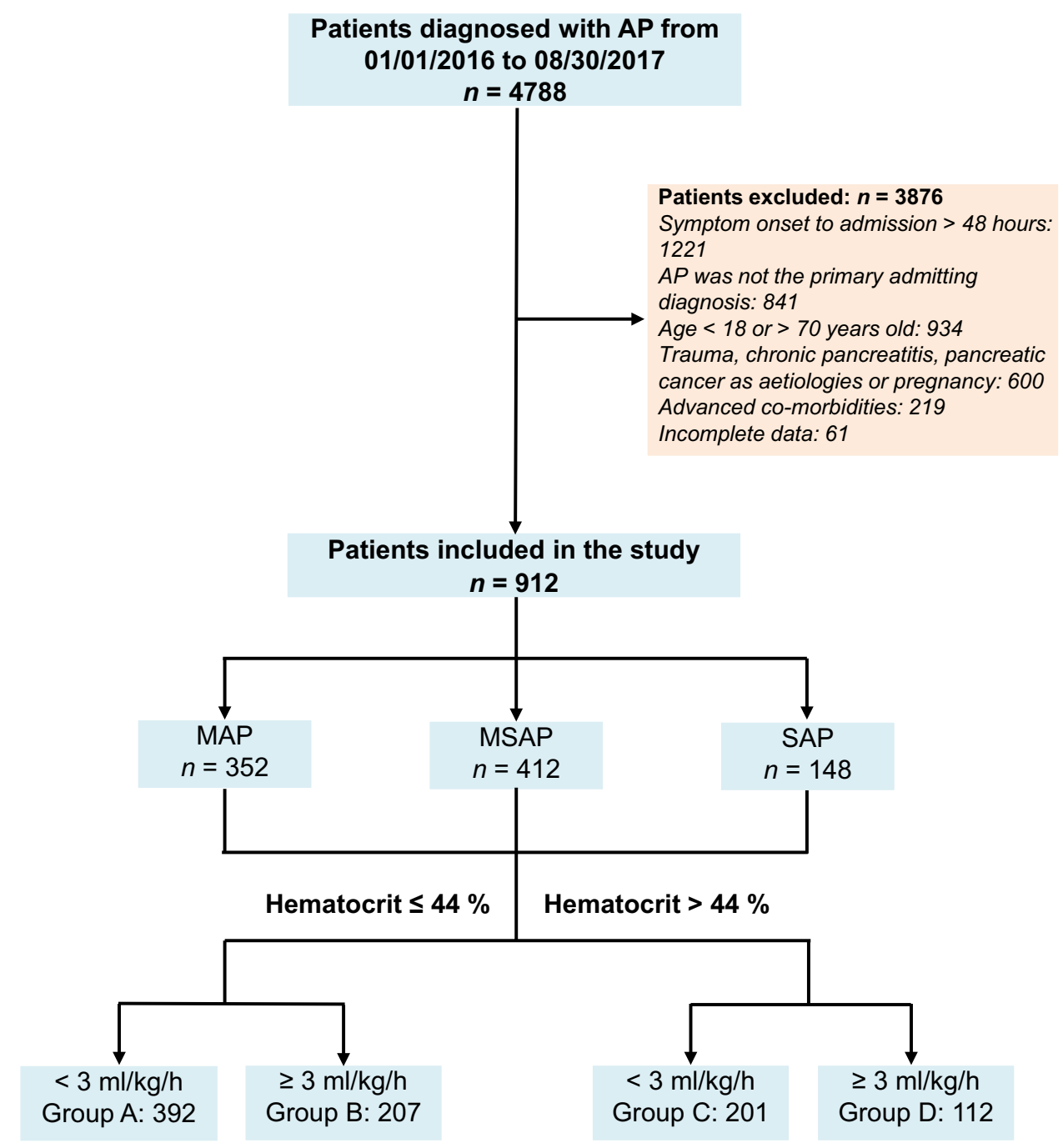

Physiology and Chronic Health Evaluation II and Sequential Organ Failure Assessment scores; all $P<0.001)$. In regard to clinical outcomes, the incidence of APFC, rate of necrosectomy, and LOS were similar between those with and without hemoconcentration. All other clinical outcomes of interest were significantly worse in the hemoconcentrated patients (Supplementary Table 2), including single persistent OF, MOF, ICU admission, respiratory support, ANC, and mortality.

\section{Clinical Outcomes of Hemoconcentrated Patients Between Different Fluid Rates}

Furthermore, there was no difference in hospital LOS in MAP (Supplementary Table 3a); there were no differences all clinical outcomes of interest in MSAP (Supplementary Table 3b). In SAP patients, there was no difference in baseline parameters (including age, gender, body mass index, Charlson comorbidity index, time to admission, etiology, and admission clinical severity scoring system) and severity of acute respiratory distress syndrome (ARDS) as per Berlin definition [37] when admitted at general wards (all $P>0.09$; Table 3); however, there was significantly higher respiratory support rate (OR 5.01, 95\% CI [1.30, 19.3], $P=0.019)$, and this was mostly attributed to higher rate of NPPV use (OR 5.96, 95\% CI [1.57, 22.6], $P=0.009$; Table 4). And there was significantly reduced predicted hospital discharge (HR $0.62[0.20,1.87], P=0.030)$, indicating significantly longer LOS in SAP (Table 4), while rate of persistent OF, MOF, local complications, necrosectomy, and mortality were similar (all $P>0.19$, Table 4).

After adjusting for different baseline parameters, subsequent analyses showed that there were no differences in clinical outcomes in all severity categories of non-hemoconcentrated patients between ESFT and ERFT (Supplementary Table 4a-c; all $P>0.15$ ).

There were no significant differences in baseline parameters of SAP patients between non-hemoconcentrated and hemoconcentrated groups in ESFT or in ERFT. None of the clinical outcomes between non-hemoconcentrated and 
Table 2 Baseline characteristics and clinical outcomes of overall included patients

\begin{tabular}{|c|c|c|c|c|c|}
\hline Parameters & Total $(n=912)$ & MAP $(n=352)$ & $\operatorname{MSAP}(n=412)$ & $\operatorname{SAP}(n=148)$ & $P$ value $^{\mathrm{a}}$ \\
\hline \multicolumn{6}{|l|}{ Demographics } \\
\hline Age, years, median (IQR) & $45(38-51)$ & $44(39-51)$ & $45(38-51)$ & $46(41-52)$ & $0.004^{\mathrm{b}}$ \\
\hline Gender, male $(\%)$ & $620(68.0)$ & $234(66.7)$ & $285(69.2)$ & $101(68.2)$ & 0.437 \\
\hline Body mass index, $\mathrm{kg} / \mathrm{m}^{2}$ median (IQR) & $25.3(23.0-27.7)$ & $25.3(22.9-27.7)$ & $24.9(22.8-27.3)$ & $26.3(24.0-28.4)$ & $<0.001^{\mathrm{b}}$ \\
\hline Charlson comorbidity index, median (IQR) & $1(0-2)$ & $2(0-2)$ & $1(0-2)$ & $1(0-2)$ & 0.593 \\
\hline \multicolumn{6}{|l|}{ Etiology } \\
\hline Biliary & $211(23.1)$ & $63(17.9)$ & $112(27.2)$ & $36(24.3)$ & $0.002^{\mathrm{c}}$ \\
\hline Hypertriglyceridemia & $335(36.7)$ & $134(38.2)$ & $140(34.0)$ & $61(41.2)$ & 0.229 \\
\hline Alcoholics & $71(7.8)$ & $31(8.9)$ & $26(6.3)$ & $14(9.5)$ & 0.241 \\
\hline Others & $295(32.3)$ & $124(35.2)$ & $134(32.5)$ & $37(25.0)$ & 0.205 \\
\hline \multicolumn{6}{|l|}{ Clinical scoring systems } \\
\hline SIRS, median (IQR) & $2(1-2)$ & $1(1-2)$ & $2(1-2)$ & $3(2-3)$ & $0.009^{\mathrm{d}}$ \\
\hline Glasgow, median (IQR) & $1(0-2)$ & $1(0-1)$ & $1(0-2)$ & $2(2-3)$ & $<0.001^{\mathrm{d}}$ \\
\hline APACHE II, median (IQR) & $4(2-6)$ & $3(2-5)$ & $4(2-6)$ & $7(5-10)$ & $<0.001^{\mathrm{d}}$ \\
\hline SOFA, median (IQR) & $0(0-1)$ & $0(0-0)$ & $0(0-1)$ & $2(0-3)$ & $<0.001^{\mathrm{d}}$ \\
\hline \multicolumn{6}{|l|}{ Clinical outcomes } \\
\hline \multicolumn{6}{|l|}{ Single persistent OF } \\
\hline Respiratory (\%) & $145(15.9)$ & 0 & 0 & $145(98.0)$ & $<0.001^{\mathrm{d}}$ \\
\hline Circulatory (\%) & $10(1.0)$ & 0 & 0 & $10(6.8)$ & $<0.001^{\mathrm{b}}$ \\
\hline Renal $(\%)$ & $22(2.4)$ & 0 & 0 & $22(14.9)$ & $<0.001^{\mathrm{b}}$ \\
\hline $\operatorname{MOF}(\%)$ & $22(2.4)$ & 0 & 0 & $22(14.9)$ & $<0.001^{\mathrm{b}}$ \\
\hline HDU/ICU admission (\%) & $157(17.2)$ & 0 & $35(8.5)$ & $122(82.4)$ & $<0.001^{\mathrm{d}}$ \\
\hline Respiratory support (\%) & $133(18.7)$ & 0 & $10(2.4)$ & $122(82.4)$ & $0.013^{\mathrm{d}}$ \\
\hline \multicolumn{6}{|l|}{ Local complication } \\
\hline Acute peripancreatic fluid collection $(\%)$ & $331(46.5)$ & 0 & $234(56.8)$ & 97 (65.5) & $<0.001^{\mathrm{d}}$ \\
\hline $\mathrm{ANC}(\%)$ & $187(20.5)$ & 0 & $128(31.3)$ & $59(39.9)$ & $<0.001^{\mathrm{d}}$ \\
\hline Necrosectomy $(\%)$ & $43(6.0)$ & 0 & $20(4.9)$ & $23(15.5)$ & $0.017^{\mathrm{d}}$ \\
\hline Mortality (\%) & $27(3.8)$ & 0 & 0 & $27(18.2)$ & $<0.001^{\mathrm{b}}$ \\
\hline Hospital LOS, median (IQR) ${ }^{\mathrm{e}}$ & $10(7-14)$ & $8(6-10)$ & $10(7-14)$ & $17(13,24)$ & $<0.001^{\mathrm{d}}$ \\
\hline
\end{tabular}

$M A P$ mild acute pancreatitis, MSAP moderately severe acute pancreatitis, SAP severe acute pancreatitis, IQR interquartile range, SIRS systemic inflammatory response syndrome, APACHE II Acute Physiology and Chronic Health Evaluation II, SOFA Sequential Organ Failure Assessment, $O F$ organ failure, $M O F$ multiple organ failure, $H D U$ high-dependency unit, $I C U$ intensive care unit, $A N C$ acute necrotic collection, $L O S$ length of stay

${ }^{a}$ Indicates $\chi^{2}$ (or Fisher's exact test) for qualitative data and Kruskal-Wallis $H$ test for quantitative data

${ }^{\mathrm{b}} P<0.05$, severe versus mild or moderate

${ }^{\mathrm{c}} P<0.05$, mild versus moderate

${ }^{\mathrm{d}} P<0.05$, between any two groups

${ }^{\mathrm{e}}$ Data from deceased patients are removed from the analysis

hemoconcentrated groups were significantly different following ESFT (Supplementary Table 5). Compared with nonhemoconcentrated patients, the hemoconcentrated patients were associated with prolonged predicted hospital discharge (HR $0.11[0.18,0.68], P=0.017$ ) following ERFT (Supplementary Table 6).

\section{Fluid Therapy Rate and Volume}

As expected and based on the definitions, there were significant differences in fluid rate between the four groups for those with and without hemoconcentration (Supplementary Fig. 3). The actual total fluid therapy volume given during the first $24 \mathrm{~h}$ was, as expected, significantly higher in patients with MSAP and SAP who received rapid fluid therapy (Supplementary Fig. 3b, c), but not in patients with MAP (Supplementary Fig. 3a). 
Table 3 Comparison of baseline parameters and ARDS level in hemoconcentrated SAP patients between different fluid rates

\begin{tabular}{|c|c|c|c|}
\hline \multirow[t]{2}{*}{ Parameters } & \multicolumn{2}{|l|}{ SAP } & \multirow[t]{2}{*}{$P$ value $^{a}$} \\
\hline & $\begin{array}{l}\text { Rate }<3 \mathrm{ml} / \mathrm{kg} / \mathrm{h} \\
n=29\end{array}$ & $\begin{array}{l}\text { Rate } \geq 3 \mathrm{ml} / \mathrm{kg} / \mathrm{h} \\
n=49\end{array}$ & \\
\hline \multicolumn{4}{|l|}{ Demographics } \\
\hline Age, years, median (IQR) & $46(41,53)$ & $46(38,52)$ & 0.580 \\
\hline Gender, male $(\%)$ & $23(79.3)$ & $36(73.5)$ & 0.599 \\
\hline Body mass index $\left(\mathrm{kg} / \mathrm{m}^{2}\right)$, median (IQR) & $26.1(23.1,28.1)$ & $26.8(24.2,30.0)$ & 0.379 \\
\hline Charlson comorbidity index, median (IQR) & $1(0-2)$ & $1(0-2)$ & 0.496 \\
\hline Time to admission (h), median (IQR) & $24(21-24)$ & $24(17-24)$ & 0.438 \\
\hline \multicolumn{4}{|l|}{ Etiology } \\
\hline Biliary $(\%)$ & $12(41.4)$ & $13(26.5)$ & 0.213 \\
\hline Hypertriglyceridemia (\%) & $8(27.6)$ & $20(40.9)$ & 0.329 \\
\hline Alcoholic (\%) & 0 & $6(12.2)$ & 0.079 \\
\hline Others $(\%)$ & $9(31.0)$ & $10(20.4)$ & 0.413 \\
\hline \multicolumn{4}{|l|}{ Clinical scoring systems } \\
\hline SIRS, median (IQR) & $3(2-3)$ & $3(2-3)$ & 0.486 \\
\hline Glasgow, median (IQR) & $2(2-3)$ & $3(2-4)$ & 0.091 \\
\hline APACHE II, median (IQR) & $8(6-11)$ & $8(7-14)$ & 0.685 \\
\hline SOFA, median (IQR) & $2(0-3)$ & $2(1-4)$ & 0.152 \\
\hline \multicolumn{4}{|l|}{ ARDS level at admission (\%) } \\
\hline Mild (200-300) & $9(33.3)$ & $9(19.6)$ & 0.188 \\
\hline Moderate (100-200) & $6(22.2)$ & $13(28.3)$ & 0.570 \\
\hline Severe $(\leq 100)$ & 0 & $2(4.3)$ & 0.272 \\
\hline
\end{tabular}

$A R D S$ acute respiratory distress syndrome, SAP severe acute pancreatitis, IQR interquartile range, SIRS systemic inflammatory response syndrome, APACHE II Acute Physiology and Chronic Health Evaluation II, SOFA Sequential Organ Failure Assessment

${ }^{a}$ Indicates $\chi^{2}$ or Fisher's exact test for qualitative data and Mann-Whitney $U$ test for quantitative data

\section{Multivariate Logistic Regression Analyses and Sensitivity Analyses}

In the multivariate logistic regression analyses, 6 (including age, time to admission, body mass index, referral, hemoconcentration, and ERFT) of 9 independent covariables were associated with persistent $\mathrm{OF}, 1$ (referral) with ANC, 4 (Charlson comorbidity index, body mass index, hemoconcentration, and ERFT) with mortality (Table 5). Admission hemoconcentration and ERFT were significantly associated with persistent OF and mortality, but not ANC (Table 5).

When restricted to patients admitted $<24 \mathrm{~h}$ after symptoms onset, hemoconcentration and ERFT were significantly associated with persistent $\mathrm{OF}$, and only hemoconcentration was significantly associated with ANC (Table 6). Mortality was removed from analysis as only 8 deaths in 41 SAP patients.

When restricted to patients admitted directly to West China Hospital, excluding those referred from other hospitals, only admission hemoconcentration and ERFT were associated with persistent $\mathrm{OF}$ and none of the other 9 covariables were significantly associated with ANC (Table 7).
Mortality was removed from analysis as only 4 deaths in 40 SAP patients.

\section{Discussion}

The key finding of this study is that hemoconcentrated SAP patients who received ERFT had an increased risk of respiratory dysfunction (requiring NPPV) and had an increased LOS. This was not found in hemoconcentrated MAP and MSAP patients. Multivariate logistic regression analyses confirmed that ERFT and hematocrit (on admission to general wards) were significantly associated with persistent $\mathrm{OF}$ and mortality, but not ANC. The important clinical implication from this study is that ERFT should be used with cautious and tailored to the patient's requirements in predicted SAP patients who remain hemoconcentrated after initial fluid therapy [38-40]. The unsolved challenge is how to reliably determine an individual patient's fluid requirements and how best to monitor the response to what is given [41].

In this study, we defined hemoconcentration as admission hematocrit level $>44 \%$, based on previous studies [28, 29, 31]. Our results show that patients with hemoconcentration 
Table 4 Comparison of clinical outcomes in hemoconcentrated SAP patients between different fluid rates

\begin{tabular}{|c|c|c|c|c|c|}
\hline \multirow[t]{2}{*}{ Clinical outcomes } & \multicolumn{2}{|l|}{ SAP } & \multirow[t]{2}{*}{$P$ value $^{\mathrm{a}}$} & \multirow[t]{2}{*}{ Estimate $(95 \% \mathrm{CI})^{\mathrm{b}}$} & \multirow[t]{2}{*}{ Adjusted $P$ value ${ }^{\mathrm{c}}$} \\
\hline & $\begin{array}{l}\text { ESFT } \\
(\mathrm{rate}<3 \mathrm{ml} / \\
\mathrm{kg} / \mathrm{h}) \\
n=29\end{array}$ & $\begin{array}{l}\text { ERFT } \\
(\text { rate } \geq 3 \mathrm{ml} / \\
\mathrm{kg} / \mathrm{h}) \\
n=49\end{array}$ & & & \\
\hline \multicolumn{6}{|l|}{ Single persistent $\mathrm{OF}$} \\
\hline Respiratory (\%) & $27(93.1)$ & $48(98.0)$ & 0.281 & $3.33(0.27,41.5)$ & 0.350 \\
\hline Circulatory $(\%)$ & $1(3.4)$ & $9(18.4)$ & 0.057 & $4.39(0.48,40.0)$ & 0.189 \\
\hline Renal (\%) & $5(17.2)$ & $11(22.4)$ & 0.582 & $0.64(0.15,2.81)$ & 0.556 \\
\hline $\operatorname{MOF}(\%)$ & $4(13.8)$ & $14(28.6)$ & 0.134 & $1.59(0.36,6.99)$ & 0.542 \\
\hline HDU/ICU admission (\%) & $23(79.3)$ & $43(87.8)$ & 0.318 & $1.89(0.52,6.89)$ & 0.333 \\
\hline Respiratory support $(\%)^{\mathrm{d}}$ & $20(69.0)$ & 45 (91.8) & 0.009 & $5.01(1.30,19.3)$ & 0.019 \\
\hline NPPV (\%) & $18(62.1)$ & $41(83.7)$ & 0.032 & $5.96(1.57,22.6)$ & 0.009 \\
\hline $\operatorname{IMV}(\%)$ & $6(20.7)$ & $15(30.6)$ & 0.340 & $1.48(0.43,5.08)$ & 0.530 \\
\hline \multicolumn{6}{|l|}{ Local complication } \\
\hline Acute peripancreatic fluid collection (\%) & $21(72.4)$ & $36(73.5)$ & 0.919 & $0.89(0.30,2.64)$ & 0.832 \\
\hline $\mathrm{ANC}(\%)$ & $13(44.8)$ & $22(44.9)$ & 0.995 & $0.99(0.37,2.50)$ & 0.925 \\
\hline Necrosectomy (\%) & $6(20.7)$ & $5(10.2)$ & 0.198 & $0.48(0.13,1.84)$ & 0.285 \\
\hline Mortality (\%) & $7(24.1)$ & $10(20.4)$ & 0.700 & $0.29(0.09,0.89)$ & 0.386 \\
\hline Hospital LOS, median (IQR) ${ }^{\mathrm{e}}$ & $17(10-22)$ & $21(13-29)$ & 0.068 & $0.62(0.20,1.87)$ & $\mathbf{0 . 0 3 0}$ \\
\hline
\end{tabular}

Bold values indicate statistical significance $P<0.05$

$S A P$ severe acute pancreatitis, $C I$ confidence interval, ESFT early slow fluid therapy, ERFT early rapid fluid therapy, $O F$ organ failure, $M O F$ multiple organ failure, $H D U$ high-dependency unit, $I C U$ intensive care unit, $N P P V$ noninvasive positive-pressure ventilation, $I M V$ invasive mechanic ventilation, $A N C$ acute necrotic collection, $L O S$ length of stay, $I Q R$ interquartile range

${ }^{a}$ Indicates $\chi^{2}$ (or Fisher's exact test) for qualitative data and Mann-Whitney $U$ test for quantitative data

${ }^{\mathrm{b}}$ Odds ratio for qualitative data and hazard ratio for quantitative data (deceased patients are considered as truncated data)

${ }^{\mathrm{c}}$ Indicates multivariate logistic regression model for qualitative data and Cox proportional hazards model quantitative data, respectively, after adjusting baseline variables from the univariate analysis with $P<0.2$

${ }^{\mathrm{d}}$ In ESFT group, 14 only had NPPV, 4 had NPPV and then IMV, 2 had IMV without prior NPPV; in ERFT group, 30 only had NPPV, 11 had NPPV and then IMV, 4 had IMV without prior NPPV

${ }^{\mathrm{e}}$ Predicted hospital discharge is used to calculate the estimate (deceased patients are considered as truncated data)

Table 5 Multivariate analyses for persistent organ failure, acute necrotic collection, and mortality in all patients

\begin{tabular}{|c|c|c|c|c|c|c|}
\hline \multirow[t]{2}{*}{ Variable } & \multicolumn{2}{|c|}{ Persistent organ failure } & \multicolumn{2}{|c|}{ Acute necrotic collection } & \multicolumn{2}{|l|}{ Mortality } \\
\hline & Estimate & $P$ & Estimate & $P$ & Estimate & $P$ \\
\hline Age $\left(\right.$ years) ${ }^{\mathrm{a}}$ & $1.04(1.01,1.05)$ & 0.003 & $1.00(0.98,1.01)$ & 0.605 & $1.04(1.00,1.09)$ & 0.079 \\
\hline Gender & $0.92(0.58,1.46)$ & 0.733 & $1.04(0.70,1.54)$ & 0.844 & $0.49(0.20,1.22)$ & 0.126 \\
\hline Charlson comorbidity index ${ }^{a}$ & $0.86(0.73,1.03)$ & 0.099 & $0.97(0.84,1.12)$ & 0.674 & $0.66(0.44,0.98)$ & $\mathbf{0 . 0 3 9}$ \\
\hline Time to admission $(\mathrm{h})^{\mathrm{a}}$ & $1.03(1.01,1.04)$ & $\mathbf{0 . 0 0 7}$ & $0.99(0.98,1.01)$ & 0.372 & $1.03(0.99,1.06)$ & 0.149 \\
\hline Body mass index $\left(\mathrm{kg} / \mathrm{m}^{2}\right)^{\mathrm{a}}$ & $1.09(1.03,1.15)$ & 0.002 & $1.04(0.99,1.08)$ & 0.134 & $1.15(1.04,1.26)$ & 0.005 \\
\hline Etiology & $0.88(0.65,1.18)$ & 0.381 & $1.10(0.87,1.39)$ & 0.425 & $0.89(0.50,1.59)$ & 0.691 \\
\hline Referral & $3.38(2.08,5.51)$ & $<0.001$ & $2.26(1.56,3.28)$ & $<0.001$ & $3.02(0.97,9.41)$ & 0.057 \\
\hline Hemoconcentration & $2.48(1.63,3.76)$ & $<0.001$ & $1.40(0.98,2.00)$ & 0.061 & $3.95(1.65,9.48)$ & 0.002 \\
\hline ERFT & $2.59(1.74,3.85)$ & $<0.001$ & $1.31(0.90,1.85)$ & 0.130 & $2.39(1.05,5.44)$ & $\mathbf{0 . 0 3 9}$ \\
\hline
\end{tabular}

Bold values indicate statistical significance $P<0.05$

at ward admission had significantly higher baseline clinical severity scores and worse clinical outcomes (persistent OF, MOF, ANC, and mortality), consistent with the published literature [28, 29, 31]. The argument for ERFT is that hemoconcentration increases blood viscosity and flow resistance [42] and that correcting this with early aggressive fluid 
Table 6 Multivariate analyses for persistent organ failure, acute necrotic collection in patients of admission $<24 \mathrm{~h}$

\begin{tabular}{|c|c|c|c|c|}
\hline \multirow[t]{2}{*}{ Variable } & \multicolumn{2}{|c|}{ Persistent organ failure } & \multicolumn{2}{|c|}{ Acute necrotic collection } \\
\hline & Estimate & $P$ & Estimate & $P$ \\
\hline Age (years) ${ }^{\mathrm{a}}$ & $1.03(0.99,1.07)$ & 0.106 & $1.00(0.97,2.11)$ & 0.760 \\
\hline Gender & $1.50(0.60,3.76)$ & 0.391 & $1.12(0.60,2.11)$ & 0.726 \\
\hline Charlson comorbidity index ${ }^{a}$ & $1.08(0.79,1.47)$ & 0.653 & $1.19(0.96,1.49)$ & 0.119 \\
\hline Time to admission $(\mathrm{h})^{\mathrm{a}}$ & $1.12(1.04,1.21)$ & 0.004 & $0.96(0.90,1.01)$ & 0.121 \\
\hline Body mass index $\left(\mathrm{kg} / \mathrm{m}^{2}\right)^{\mathrm{a}}$ & $1.05(0.95,1.16)$ & 0.328 & $1.04(0.97,1.13)$ & 0.275 \\
\hline Etiology & $1.26(0.74,2.16)$ & 0.392 & $1.43(0.97,2.11)$ & 0.071 \\
\hline Referral & $2.03(0.96,4.28)$ & 0.063 & $2.11(1.23,3.63)$ & 0.007 \\
\hline Hemoconcentration & $5.74(2.63,12.5)$ & $<0.001$ & $1.77(1.01,3.09)$ & 0.045 \\
\hline ERFT & $3.36(1.58,7.14)$ & 0.002 & $1.44(0.82,2.53)$ & 0.202 \\
\hline
\end{tabular}

Bold values indicate statistical significance $P<0.05$

\begin{tabular}{|c|c|c|c|c|}
\hline \multirow[t]{2}{*}{ Variable } & \multicolumn{2}{|c|}{ Persistent organ failure } & \multicolumn{2}{|c|}{ Acute necrotic collection } \\
\hline & Estimate & $P$ & Estimate & $P$ \\
\hline Age $(\text { years })^{\mathrm{a}}$ & $1.01(0.97,1.05)$ & 0.836 & $0.98(0.95,1.01)$ & 0.286 \\
\hline Gender & $0.44(0.16,1.22)$ & 0.116 & $1.01(0.50,2.04)$ & 0.974 \\
\hline Charlson comorbidity index ${ }^{a}$ & $0.98(0.69,1.39)$ & 0.898 & $1.11(0.87,1.41)$ & 0.418 \\
\hline Time to admission $(\mathrm{h})^{\mathrm{a}}$ & $1.02(0.97,1.06)$ & 0.471 & $0.99(0.96,1.02)$ & 0.592 \\
\hline Body mass index $\left(\mathrm{kg} / \mathrm{m}^{2}\right)^{\mathrm{a}}$ & $1.09(0.96,1.24)$ & 0.177 & $1.04(0.95,1.13)$ & 0.419 \\
\hline Etiology & $1.13(0.59,2.18)$ & 0.715 & $1.31(0.85,2.03)$ & 0.225 \\
\hline Hemoconcentration & $2.95(1.51,7.54)$ & 0.024 & $1.13(0.59,2.20)$ & 0.709 \\
\hline ERFT & $3.12(1.28,7.58)$ & 0.012 & $1.54(0.81,2.92)$ & 0.187 \\
\hline
\end{tabular}

Age, gender (male vs. female), Charlson comorbidity index, time to admission, body mass index, etiology (hypertriglyceridemia, biliary, alcoholic, others), transfer (yes vs. no), hemoconcentration (yes, hematocrit $>44 \%$ vs. no, $\leq 44 \%$ ), fluid rate (fast, $\geq 3 \mathrm{ml} / \mathrm{kg} / \mathrm{h}$ vs. slow, $<3 \mathrm{ml} / \mathrm{kg} / \mathrm{h}$ ) were included in multivariate logistic regression analysis

Bold values indicate statistical significance $P<0.05$

$E R F T$ early rapid fluid therapy

${ }^{\mathrm{a} C}$ Continuous variable therapy will improve organ perfusion (e.g., pancreas, lung, kidneys) and clinical outcomes of AP [28, 29]. Although oxygen-carrying capacity of the blood is reduced during hemodilution by fluid therapy, there are limits to the compensatory mechanisms (increased cardiac output and tissue oxygen extraction) with extreme hemodilution [42]. Experimental studies have suggested that both hemoconcentration and rapid hemodilution are associated with increased severity of the underlying diseases [43, 44]. A randomized clinical trial has demonstrated that rapid hemodilution was associated with increased sepsis and mortality in patients with predicted SAP [16]. The findings of the present study are consistent with this trial as ERFT in hemoconcentrated SAP patients was associated with a significant increased respiratory dysfunction (and need for NPPV) and LOS even after adjustment for differences in baseline clinical severity scores (including SIRS, Glasgow, Acute Physiology and
Chronic Health Evaluation II and Sequential Organ Failure Assessment scores). The most common OF is ARDS, and the onset of persistent OF is often early (with $60 \%$ in the first day of admission) [2, 45, 46]. Given that ARDS is already present or is likely to occur in the majority of patients within the first $24 \mathrm{~h}$, ERFT might be expected to worsen the severity of ARDS. Patients who are hypovolemic and without significant capillary leak will likely benefit from early and rapid fluid resuscitation, but by the time capillary leak has developed (which contributes to ARDS development) additional fluids will increase interstitial fluid volume (edema) and cause worsening ARDS. In West China Hospital, it is established practice to use NPPV to manage patients with early ARDS or at risk of ARDS [34], and it is administered on the basis of standard criteria. In this study, it was found that there was an increased requirement for NPPV in patients 
receiving ERFT (compared with ESFT) even though the ARDS severity was comparable between these two groups.

By avoiding invasive ventilation in the majority of patients, NPPV can be an effective way to improve gas exchange and avoid complications associated with endotracheal intubation and mechanical ventilation in selected patients with ARDS [47-49]. It may be the most effective option for the initial treatment for all categories of ARDS secondary to AP as long as no contra-indication exists [34].

In hemoconcentrated SAP patients of our study, there was no difference in severity of ARDS [37] at ward admission between those receiving ERFT and ESFT. But there was a significantly higher rate of NPPV use in the ERFT group. This difference may be due to a significantly faster rate of fluid therapy during the first $6 \mathrm{~h}$ and/or a higher total fluid volume during $24 \mathrm{~h}$ in the ERFT group (Supplementary Fig. 3). The finding is consistent with studies which showed that rapid and high volume fluid therapy increases the risk of pulmonary edema and exacerbates respiratory failure in AP [13, 24]. Our study has several limitations. The fluid management data were collected retrospectively, and there was a lack of data for fluid given prior to admission to the general ward. The hematocrit prior to fluid therapy in ED was also not available. However, the prospective data we have collected indicate that a mean of approximately $1000 \mathrm{ml}$ fluid was given in the ED prior to general ward admission. Another potential confounder in this study was that patients with hemoconcentration may not have received enough fluid, despite no difference between hemoconcentrated and non-hemoconcentrated patients in regard to time to admission and volume of fluid given. Hemoconcentrated patients had more severe disease and were therefore at greater risk of respiratory dysfunction such that ERFT may be just an association and not a cause. With this study design, it is not possible to absolutely prove that ERFT caused an increased requirement of NPPV. Lastly, this was a single-center cohort composed by high proportion of patients with a hyperlipidemia as etiology of AP [2, 35, 36], which may limit the generalizability of our findings.

In conclusion, this study has shown that ERFT in hemoconcentrated patients with SAP patients is associated with increased use of NPPV and longer LOS. This was not the case in hemoconcentrated patients with MAP and MSAP or in non-hemoconcentrated patients in all three grades of severity. Because of the risks identified in this study, a better understanding of why ERFT is associated with worse clinical outcomes in hemoconcentrated patients with SAP is important. Appropriately designed randomized clinical trials are urgently needed to determine how fluid therapy should be given in patients with predicted severe disease and hemoconcentration.
Acknowledgments These authors thank all the staff from pancreas multidisciplinary team at West China Hospital for their continuous support. This study was supported by NZ-China Strategic Research Alliance 2016 Award (Ministry of Science and Technology, China (Grant No. 2016YFE0101800), QX, TJ, WH, XY, LD, and JG; Health Research Council, New Zealand, AP and JAW), and National Institute for Health Research Senior Investigator Award (RS).

Author's contribution WH, QX, JAW, and TJ were involved in the concept and design; LL, TJ, SW, RZ, NS, ZL, KJ, JG, TL, LD, and XY acquired the data; $\mathrm{LL}$ and $\mathrm{PZ}$ were involved in the statistical analysis and interpretation of data; LL and WH drafted the manuscript; AP, VKS, RS, WH, QX, and JAW revised the manuscript; WH, QX, and JAW obtained the funding; all authors have approved the final version of manuscript submitted.

\section{Compliance with Ethical Standards}

Conflict of interest RS has provided consultancy to Abbot (Mylan); no further support from any organization for the submitted work; no financial relationships with any organizations that might have had an interest in the submitted work in the previous 3 years; no other relationship or activities that could appear to have influenced the submitted work.

Open Access This article is licensed under a Creative Commons Attribution-NonCommercial 4.0 International License, which permits any non-commercial use, sharing, adaptation, distribution and reproduction in any medium or format, as long as you give appropriate credit to the original author(s) and the source, provide a link to the Creative Commons licence, and indicate if changes were made. The images or other third party material in this article are included in the article's Creative Commons licence, unless indicated otherwise in a credit line to the material. If material is not included in the article's Creative Commons licence and your intended use is not permitted by statutory regulation or exceeds the permitted use, you will need to obtain permission directly from the copyright holder.To view a copy of this licence, visit http://creativecommons.org/licenses/by-nc/4.0/.

\section{References}

1. Banks PA, Bollen TL, Dervenis C, et al. Classification of acute pancreatitis-2012: revision of the Atlanta classification and definitions by international consensus. Gut. 2013;62:102-111.

2. Shi N, Liu T, de la Iglesia D, et al. Duration of organ failure impacts mortality in acute pancreatitis. Gut 2019, gutjnl-2019-318241.

3. Parniczky A, Kui B, Szentesi A, et al. Prospective, multicentre, nationwide clinical data from 600 cases of acute pancreatitis. PLoS One. 2016;11:e0165309.

4. Moggia E, Koti R, Belgaumkar AP, et al. Pharmacological interventions for acute pancreatitis. Cochrane Database Syst Rev. 2017;4:CD011384.

5. Tenner S, Baillie J, DeWitt J, et al. American College of Gastroenterology guideline: management of acute pancreatitis. Am J Gastroenterol. 2013;108:1400-1416.

6. Working Group IAPAPAAPG. IAP/APA evidence-based guidelines for the management of acute pancreatitis. Pancreatology. 2013;13:e1-e15.

7. Haydock MD, Mittal A, Wilms HR, et al. Fluid therapy in acute pancreatitis: anybody's guess. Ann Surg. 2013;257:182-188. 
8. Warndorf MG, Kurtzman JT, Bartel MJ, et al. Early fluid resuscitation reduces morbidity among patients with acute pancreatitis. Clin Gastroenterol Hepatol. 2011;9:705-709.

9. Dimagno MJ, Wamsteker EJ, Rizk RS, et al. A combined paging alert and web-based instrument alters clinician behavior and shortens hospital length of stay in acute pancreatitis. Am J Gastroenterol. 2014;109:306-315.

10. Singh VK, Gardner TB, Papachristou GI, et al. An international multicenter study of early intravenous fluid administration and outcome in acute pancreatitis. United European Gastroenterol J. 2017;5:491-498.

11. Buxbaum JL, Quezada M, Da B, et al. Early aggressive hydration hastens clinical improvement in mild acute pancreatitis. Am J Gastroenterol. 2017;112:797-803.

12. Weitz G, Woitalla J, Wellhoner P, et al. Detrimental effect of high volume fluid administration in acute pancreatitis: a retrospective analysis of 391 patients. Pancreatology. 2014;14:478-483.

13. de Madaria E, Soler-Sala G, Sanchez-Paya J, et al. Influence of fluid therapy on the prognosis of acute pancreatitis: a prospective cohort study. Am J Gastroenterol. 2011;106:1843-1850.

14. Ke L, Ni HB, Sun JK, et al. Risk factors and outcome of intraabdominal hypertension in patients with severe acute pancreatitis. World J Surg. 2012;36:171-178.

15. Mao EQ, Tang YQ, Fei J, et al. Fluid therapy for severe acute pancreatitis in acute response stage. Chin Med J (Engl). 2009;122:169-173.

16. Mao EQ, Fei J, Peng YB, et al. Rapid hemodilution is associated with increased sepsis and mortality among patients with severe acute pancreatitis. Chin Med J (Engl). 2010;123:1639-1644.

17. Yokoe M, Takada T, Mayumi T, et al. Japanese guidelines for the management of acute pancreatitis: Japanese Guidelines 2015. J Hepatobiliary Pancreat Sci. 2015;22:405-432.

18. Crockett SD, Wani S, Gardner TB, et al. American Gastroenterological Association Institute Guideline on initial management of acute pancreatitis. Gastroenterology. 2018;154:1096-1101.

19. Rivers E, Nguyen B, Havstad S, et al. Early goal-directed therapy in the treatment of severe sepsis and septic shock. $N$ Engl J Med. 2001;345:1368-1377.

20. Investigators A, Group ACT, Peake SL, et al. Goal-directed resuscitation for patients with early septic shock. $N$ Engl J Med. 2014;371:1496-506.

21. Pro CI, Yealy DM, Kellum JA, et al. A randomized trial of protocol-based care for early septic shock. $N$ Engl J Med. 2014;370:1683-1693.

22. Mouncey PR, Osborn TM, Power GS, et al. Trial of early, goal-directed resuscitation for septic shock. $N$ Engl J Med. 2015;372:1301-1311

23. Wilms H, Mittal A, Haydock MD, et al. A systematic review of goal directed fluid therapy: rating of evidence for goals and monitoring methods. J Crit Care. 2014;29:204-209.

24. Jin T, Jiang K, Deng L, et al. Response and outcome from fluid resuscitation in acute pancreatitis: a prospective cohort study. HPB (Oxford). 2018;20:1082-1091.

25. Haydock MD, Mittal A, van den Heever M, et al. National survey of fluid therapy in acute pancreatitis: current practice lacks a sound evidence base. World J Surg. 2013;37:2428-2435.

26. Gray SH, Rosenman LD. Acute pancreatitis. The significance of hemoconcentration at admission to the hospital. Arch Surg. 1965;91:485-489.

27. Baillargeon JD, Orav J, Ramagopal V, et al. Hemoconcentration as an early risk factor for necrotizing pancreatitis. Am J Gastroenterol. 1998;93:2130-2134.
28. Brown A, Orav J, Banks PA. Hemoconcentration is an early marker for organ failure and necrotizing pancreatitis. Pancreas. 2000;20:367-372.

29. Brown A, Baillargeon JD, Hughes MD, et al. Can fluid resuscitation prevent pancreatic necrosis in severe acute pancreatitis? Pancreatology. 2002;2:104-107.

30. Wu BU, Johannes RS, Conwell DL, et al. Early hemoconcentration predicts increased mortality only among transferred patients with acute pancreatitis. Pancreatology. 2009;9:639-643.

31. Koutroumpakis E, Wu BU, Bakker OJ, et al. Admission hematocrit and rise in blood urea nitrogen at $24 \mathrm{~h}$ outperform other laboratory markers in predicting persistent organ failure and pancreatic necrosis in acute pancreatitis: a post hoc analysis of three large prospective databases. Am J Gastroenterol. 2015;110:1707-1716.

32. von Elm E, Altman DG, Egger M, et al. Strengthening the Reporting of Observational Studies in Epidemiology (STROBE) statement: guidelines for reporting observational studies. $B M J$. 2007;335:806-808.

33. Cecconi M, De Backer D, Antonelli M, et al. Consensus on circulatory shock and hemodynamic monitoring. Task force of the European Society of Intensive Care Medicine. Intensive Care Med. 2014;40:1795-1815.

34. Zhao X, Huang W, Li J, et al. Noninvasive positive-pressure ventilation in acute respiratory distress syndrome in patients with acute pancreatitis: a retrospective cohort study. Pancreas. 2016;45:58-63.

35. Zhang R, Deng L, Jin T, et al. Hypertriglyceridaemia-associated acute pancreatitis: diagnosis and impact on severity. $H P B$ (Oxford). 2019;21:1240-1249.

36. Mukherjee R, Nunes Q, Huang W, et al. Precision medicine for acute pancreatitis: current status and future opportunities. Precision Clinical Medicine. 2019;2:81-86.

37. Force ADT, Ranieri VM, Rubenfeld GD, et al. Acute respiratory distress syndrome: The Berlin Definition. JAMA. 2012;307:2526-2533.

38. Beitler JR, Schoenfeld DA, Thompson BT. Preventing ARDS: Progress, promise, and pitfalls. Chest. 2014;146:1102-1113.

39. Hashimoto S, Sanui M, Egi M, et al. The clinical practice guideline for the management of ARDS in Japan. J Intensive Care. 2017;5:50.

40. Wu BU. Editorial: fluid resuscitation in acute pancreatitis: Striking the right balance. Am J Gastroenterol. 2011;106:1851-1852.

41. Stigliano S, Sternby H, de Madaria E, et al. Early management of acute pancreatitis: A review of the best evidence. Dig Liver Dis. 2017;49:585-594.

42. Schumacker PT, Cain SM. The concept of a critical oxygen delivery. Intensive Care Med. 1987;13:223-229.

43. Cabrales P, Tsai AG, Frangos JA, et al. Oxygen delivery and consumption in the microcirculation after extreme hemodilution with perfluorocarbons. Am J Physiol Heart Circ Physiol. 2004;287:H320-H330.

44. Morariu AM, Maathuis MH, Asgeirsdottir SA, et al. Acute isovolemic hemodilution triggers proinflammatory and procoagulatory endothelial activation in vital organs: Role of erythrocyte aggregation. Microcirculation. 2006;13:397-409.

45. Liu T, Huang W, Szatmary P, et al. Accuracy of circulating histones in predicting persistent organ failure and mortality in patients with acute pancreatitis. Br J Surg. 2017;104:1215-1225.

46. Schepers NJ, Bakker OJ, Besselink MG, et al. Impact of characteristics of organ failure and infected necrosis on mortality in necrotising pancreatitis. Gut. 2019;68:1044-1051. 
47. Wang YM, Tao YH. Mechanical ventilation in acute respiratory distress syndrome. Zhongguo Dang Dai Er Ke Za Zhi. 2013;15:496-501.

48. Young D, Lamb SE, Shah S, et al. High-frequency oscillation for acute respiratory distress syndrome. $N$ Engl J Med. 2013;368:806-813.

49. Hess DR. The evidence for noninvasive positive-pressure ventilation in the care of patients in acute respiratory failure: a systematic review of the literature. Respir Care. 2004;49:810-829.
Publisher's Note Springer Nature remains neutral with regard to jurisdictional claims in published maps and institutional affiliations. 